\title{
UN ANÁLISIS PARCIAL A LA PRODUCCIÓN ACADÉMICA EN EL CAMPO PROFESIONAL DE LA EDUCACIÓN FÍSICA EN COLOMBIA
}

\author{
LIC. LEÓN JAIME URREGO DUQUE \\ Licenciado en Educación Física y Profesor Auxiliar del Instituto de \\ Educación Física de La Universidad de Antioquia (Medellín - Antioquia - Colombia) \\ E-mail: leonurrego@gmail.com
}

\begin{abstract}
RESUMEN
El artículo presenta avances de la investigación acerca la producción académica en la Educación física en Colombia entre los años 1990 y 2006, en los que se identifican rasgos académicos, formas comunicativas, actores y escenarios implicados en dicho campo profesional. Se implemento como métodos el análisis de discurso documental y las entrevistas en profundidad buscando abordar el cuestionamiento por la identidad profesional y la interacción de saberes, teorías, métodos y conocimientos que configuran y constituyen esta parcela académica.
\end{abstract}

PALABRAS CLAVE: Educación física, campo profesional, identidad profesional, producción académica. 


\section{INTRODUCCIÓN Y CONTEXTUALIZACIÓN DEL PROBLEMA ESTUDIADO}

¿Por qué tomar la producción académica de la Educación física como problema de investigación? Muchas disciplinas, ciencias y profesiones someten a revisión sus problemas, conceptos, teorías y actuaciones; esto se debe a la necesidad permanente de orientar transformaciones y ajustes o, de reconocer y ratificar sus logros. Este documento presenta resultados parciales del "Análisis a la producción académica de la Educación física en Colombia 1990-2006"', investigación que revisa discursos y prácticas de producción de conocimiento en el campo profesional. El propósito es identificar problemas (temáticas, sub-temáticas), procedimientos (formas de comunicar), destinatarios (potenciales beneficiarios) y objetivos de dicha producción para contribuir, en alguna medida, a la investigación que permita conocer y comprender el funcionamiento del campo; en su conjunto el trabajo busca hacer una interpretación de la identidad y la lógica que opera en la profesión, específicamente en el contexto colombiano.

La preocupación por revelar la identidad o la naturaleza de la Educación física ha tomado diversas formas y sentidos que se manifiestan en búsquedas para establecer sus dimensiones como ciencia, disciplina, saber, técnica, ocupación, campo, etcétera; en otras palabras, podría decirse que dicha inquietud obedece a una pretensión de posicionamiento (CRISORIO, 2003). Este interés ha estado presente en el devenir histórico de la profesión, no se trata de un cuestionamiento contemporáneo, sin embargo, en las últimas décadas se experimenta una proliferación de discursos y prácticas que buscan definir, delimitar y especificar todo el universo de problemas, objetos, métodos y actuaciones que circulan en la profesión en cuestión.

Este tipo de inquietudes recurre a argumentaciones que incluyen las reflexiones semánticas, las revisiones epistemológicas, los estudios ontológicos o incluso explicaciones de mayor complejidad. Las delimitaciones producidas en los estudios semánticos se pueden percibir en trabajos que encauzan el análisis a partir proponer o refutar las significaciones posibles del término "Educación física" y los alcances de cada acepción en el campo profesional; se trata de una exposición con base en la sinonimia y la asimilación, la diferencia y contradicción con otros sentidos y términos. Este tipo de explicaciones busca consensos conceptuales que permitan ordenar, orientar la formación, la actuación y en cierto modo la producción.

I. Este artículo es un producto investigativo de los estudios doctorales que he venido desarrollando en la Universidad de Valladolid (España) en "Didáctica de la Educación Artística: educación plástica, educación musical y Educación física" Bajo la tutoría de los profesores José Ignacio Barbero González y Nicolás Julio Bores Calle, Estudios que han contado con apoyos de la Fundación Carolina (España) y la Universidad de Antioquia (Colombia). 
Las distinciones epistemológicas han pretendido guiar los rumbos que deberían tomar los estudios y la investigación, se trata del análisis de los diversos objetos y métodos que pueden presentarse en el campo profesional. En esta línea existen trabajos que buscan naturalizar un quehacer científico en la Educación física, asociarla y adscribirla como dependiente de otras ciencias o saberes para dotarla de mejores herramientas académicas, buscando acceder a un mejor estatus científico o profesional que permita alcanzar la verdad objetiva como característica propia de las comunidades académicas reconocidas en la que es hoy llamada la sociedad del conocimiento.

Otro viso que pueden tomar las explicaciones de la profesión pretende aclarar la procedencia ontológica de la Educación física buscando el ser y las propiedades trascendentales de la profesión, acá podrían agruparse todos los trabajos que hacen un despliegue de paradigmas (nociones de hombre, ser, mundo, existencia, cuerpo, etcétera) sobre los cuales se puedan apoyar las ideas y las prácticas de los profesionales. Estas explicaciones apelan al desmontaje de tradiciones (añejas, contradictorias o sin fundamento) y se proponen paso a paso ir eliminando los obstáculos teóricos para acceder a una comprensión originaria de la profesión que permita cubrir la falta de fundamento, otorgar y producir nuevos horizontes profesionales.

Estas tres formas de explicar y definir la profesión participan con contundencia en los debates acerca de la identidad y la naturaleza de la Educación física, incluso son protagonistas de las reformas institucionales que experimenta el campo debido a que procuran en gran medida formular e instaurar un deber ser, este tipo de explicaciones del campo sugieren imponer lo que llama Bourdieu el "principio sistemático de error que es la tentación de la visión soberana” (BOURDIEU, 2002, p. 13-22).

Con este trabajo se busca interpretar las realizaciones de quienes participan históricamente de la construcción de la Educación física y de este modo aportar a la comprensión de su identidad desde otra perspectiva diferente a las tres antes mencionadas, dando un giro que permita prescindir de la visión soberana, procurando evitar las grandes categorías, los paradigmas clásicos y mejor privilegiando la interpretación de los discursos de los protagonistas del campo profesional. Un punto de partida para dimensionar y reconocer la profesión podría fundarse con la búsqueda de respuestas a los siguientes interrogantes: ¿Cuáles son los problemas que orientan los discursos y las prácticas de producción de conocimiento en la Educación física en Colombia? ¿Qué tipo de tratamiento se da a dichos problemas? ¿Cuáles son los fines y los beneficiarios de la producción de conocimiento?

Al abordar las anteriores preguntas se busca: (a) mostrar e ilustrar el desarrollo del campo profesional de la Educación física en el período comprendido entre 1990 y 2006; (b) identificar los sub-campos y las tendencias que se han 
formado señalando diferencias y fronteras, (b- I) caracterizar la diversidad temática, de enfoques o paradigmas, de referentes, de propósitos e intenciones que dotan de una relativa unidad a los distintos sub-campos; (b-2) detectar la configuración de redes (más o menos institucionalizadas) en la producción de conocimientos, y la variedad de los destinatarios de dichos conocimientos.

Es importante anotar que las ideas conductoras de el trabajo coinciden parcialmente con las de Crisorio, quien propone indagar por la identidad de la profesión en sus "prácticas reales" y anota que:

El proyecto de una teoría de la práctica de la Educación física empieza por subrayar las múltiples dimensiones del saber y de la práctica. En lugar de seccionar el saber y jerarquizarlo, dividiéndolo, por ejemplo, en conocimiento puro y aplicado, procura recuperar los caminos de ida y vuelta que comunican los saberes teóricos, técnicos y prácticos articulados en la acción (CRISORIO, 2003, p. 23-24).

Así pues, esta toma de posición y las elecciones de enfoque epistemológico (conceptos, nociones, teorías) y metodológico demando una revisión de algunas ideas generales que aparentemente gozan de claridad suficiente, pero dado el sentido y propósito de esta indagación, requieren puntualidad conceptual para nociones como conocimiento, ciencia, legitimidad, verdad, profesión. Se procura tomar distancia de los tipos de análisis señalados anteriormente (semántico, epistemológico, ontológico) buscando una mirada que acepte la inclusión y el reconocimiento de todo el panorama de saberes y formas que se manifiestan en la Educación física, en procura de evitar una mirada discriminativa hacia los aprioris y juicios de valor que operan tradicionalmente en los espacios universitarios.

Por lo anterior se hace necesario revisar el marco conceptual partiendo de la ya clásica idea de sociedad del conocimiento², porque representa las características de búsqueda y materialización de los ideales de modernización, innovación y progreso tecnológico que son los que han venido promoviendo e imponiendo unas formas contemporáneas de conocer y al tiempo van discriminando otras. El marco para la realización de dichos ideales lo constituye el conocimiento producido por medio de la ciencia, entendida clásicamente como el proceso metódico y sistemático que produce conocimiento claro, objetivo, evidente, neutral y verificable (también conocida como ciencia empírica).

Una de las consecuencias del posicionamiento de la ciencia como modo preferencial o privilegiado de acceder y producir conocimiento es la instauración

2. Para ampliar la noción de Sociedad de conocimiento sus alcances e implicaciones, ver Martín-Barbero (2002), Olive (2006). 
de sistemas de legitimación y validez que generan modos particulares de percibir y aprehender. Es decir que la ciencia ha logrado constituir estructuras que tienen influencia y determinación cultural o social, las cuales se propagan en todas las instituciones y las que se ocupan de la Educación física no son excepción, vemos por ejemplo que algunas prácticas y tendencias en el campo gozan de mayor reconocimiento u status en función de su proximidad con métodos científicos y otras prácticas son caracterizadas como "poco solidas, sin seriedad, e irrelevantes..." en la medida que se aproximan a otras formas y métodos diferentes a los empírico-científicos.

No obstante la noción de ciencia ha experimentado un fenómeno expansivo, y si se quiere transformativo, que no solo comprende a

Las ciencias desarrolladas o "maduras", hoy también acoge las tentativas de construir teorías, los modos de surgimiento de nuevas ciencias, la determinación de los ámbitos susceptibles de investigación, las razones de aceptación o rechazo de esos ámbitos. (ROSSI, 1990, p. 46)

En los últimos 50 años la investigación histórica y sociológica ha enseñado que el conocimiento se debe a unos presupuestos y condicionamientos culturales, la ciencia es una producción colectiva que depende de las convicciones y las estructuras sociológicas de los individuos (FLECK, 1986). Ahora bien, a la imagen clásica de ciencia se le contrapone la visión que admite los condicionamientos directos e indirectos de las relaciones sociales e institucionales $y$, de las agrupaciones humanas que ejercen presión material y simbólica en el conocimiento y sus modos de producirse. Por tanto, la validez y la objetividad no solo están determinadas por la claridad de los procedimientos metodológicos empleados para crear el conocimiento, sino que se encuentran afectadas por el sistema de las relaciones sociales en las cuales se realiza la creación (BOURDIEU, 2002b).

Las anteriores reseñas conducen a contextualizar el sentido que ha orientado la investigación acá propuesta, se trata de interpretar las identidades que puede asumir la Educación fisica a partir de los discursos de los protagonístas buscando tomar en cuenta el arraigo de conocimiento en la textura social (MANNHEIM, 1987), las relaciones, las posiciones, los mecanismos y los actores sociales que han ido constituyendo y configurando la profesión estudiada.

\section{METODOLOGÍA Y PROCEDIMIENTOS}

Los problemas y datos que se abordan son tratados en dos modalidades de la investigación cualitativa, una que se refiere a la Investigación Bibliográfica y la otra que remite a la indagación por medio de la Conversación Conceptual (o entrevistas en profundidad). 
La modalidad de Investigación Bibliográfica aborda el estudio de los discursos escritos, buscando comprender la realidad que es expresada, se trata de develar el sentido de la estructura que subyacen en los textos y conocer el alcance que tienen a nivel social, cultural o personal. Como instrumento para la recolección de datos en esta modalidad se ha diseñado una ficha bibliográfica para conservar los datos de manera sistemática, operaciones que servirán para el análisis descriptivo de los datos y facilitaran su consulta permanente. La elaboración de una ficha para registrar los datos implica realizar observaciones previas o iniciales de la fuente documental objeto de investigación para tener algunas ideas sobre el tipo de material a consignar. A continuación se presenta un ejemplo de la ficha:

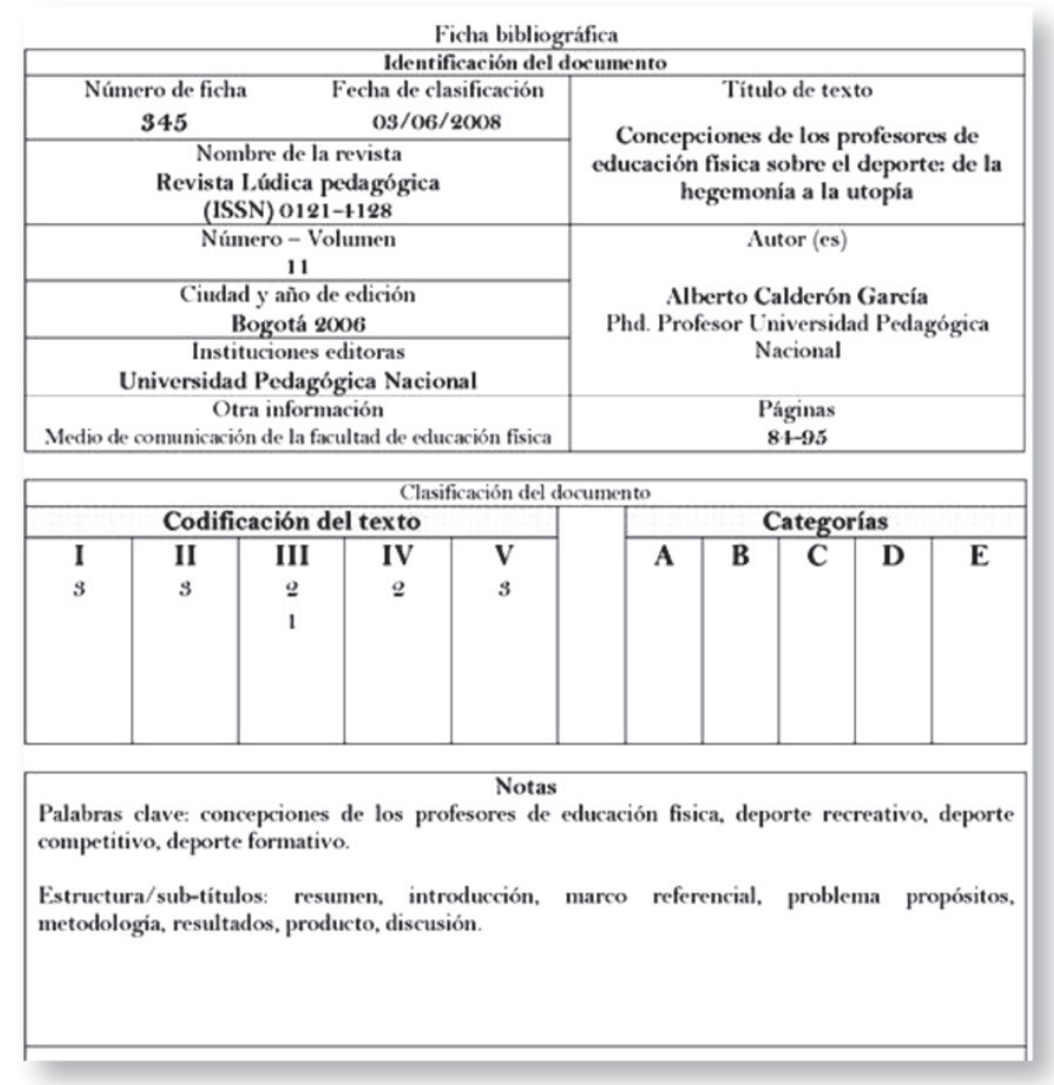

Figura I. Ejemplo de ficha bibliográfica.

La ficha contiene tres espacios, un primer espacio con datos de identificación del documento, un segundo espacio para la clasificación del documento a partir de códigos y categorías y un tercer espacio para adjuntar notas y observaciones sobre el documento. Se implemento un tipo de análisis denominado Análisis de Contenido, consistente en la lectura "técnica o sistemática" de documentos escritos para interpretar aspectos que no están en la superficie textual.

El primer momento metodológico consiste en elaboración de las fichas que inicia con la lectura de los documentos, la codificación y clasificación en catego- 
rías que la lectura texto arrojando. Un segundo momento en el análisis consiste en realizar una primera revisión cuantitativa que orienta el análisis cualitativo. tercer momento del análisis requiere que las fichas sean agrupadas siguiendo sus regularidades, buscando reunirlas primero por categorías, luego por sub-temáticas, después por niveles (teóricas, prácticas, etcétera), y por las demás posibilidades de agrupación que emerjan de la lectura de los propios textos.

En el primer párrafo del presente apartado "Metodología y procedimientos" se menciona el uso de dos modalidades metodológicas y se esbozo la que se refiere al Estudio Bibliográfico; y ahora solo resta aclarar que con la segunda modalidad denominada La Conversación Conceptual se han atendido los discursos orales o los relatos directos que se producen a partir de una conversación o entrevista en profundidad sostenida entre el investigador y actores protagónicos ${ }^{3}$ del campo profesional de la Educación física. Esta investigación emplea una entrevista no estructurada o flexible, de tipo individual y mono-temática; se ha requerido la elaboración de un guión de entrevista adecuado a su tipo y el análisis de las mismas ha seguido pasos sistemáticos como estructuración, teorización y la elaboración de un texto para presentarlas y comunicarlas (RUIZ OLABUÉNAGA, 2003). No se ampliara la explicación de la Conversación conceptual debido a que la difusión de dichas fuentes y análisis se proyecta para otra publicación, acá solo se han empleado fragmentos de las Entrevistas en Profundidad para dar mayor consistencia a las fuentes empleadas.

Con el empleo de estas dos variaciones o complementaciones metodológicas (Investigación Bibliográfica y Conversación Conceptual) se quiere desplegar una estrategia que permita presentar la realidad desde dos perspectivas, este tipo de estrategias de indagación con dos fuentes de datos es denominada por Carrasco y Calderero Hernández (2007, p. I 07) como "métodos solapados" que permiten recoger información sobre un mismo problema o hecho de forma que se abarque más el panorama del tema. Con la estrategia de solapamiento de métodos se sugiere una dependencia

3. Son actores que participan de la producción en la Educación Física; ellos perciben, experimentan, crean, modifican y se asumen en la realidad de la profesión en la que se hallan inmersos. La muestra de datos utilizada para la modalidad de investigación cualitativa de Conversación Conceptual consta de los relatos transcriptos de ocho informantes que representan los casos (categorías o unidades significativas) existentes en el universo de datos que es objeto de la Investigación Bibliográfica. Los criterios que determinaron la selección de los informantes se basan principalmente en su producción bibliográfica, es decir que las personas fueron elegidas con base en la lectura de los artículos revisados previamente en el estudio bibliográfico. Se eligieron dos informantes por cada categoría o Unidad Significativa, una persona novel (no más de dos publicaciones) y otra veterana (con más de 5 publicaciones y con reconocimiento académico en varias ciudades, participación en investigaciones y eventos), de este modo obtuve una visión sólida, elaborada y con reconocimiento pero también, con la participación y aporte de observaciones menos elaboradas. Decidí cambiar los nombres reales de los informantes debido a que en algunas ocasiones sus posiciones frente a otras visiones son radicales, especialmente los novatos; no obstante los relatos de las personas veteranas dejan intuir la autoría de los mismos.

Rev. Bras. Ciênc. Esporte, Florianópolis, v. 34, n. I, p. 127-148, jan./mar. 2012 
entre las fuentes orales y escritas, y se intenta incrementar el nivel de fiabilidad de la información recolectada (RUIZ OLABUÉNAGA, 2003).

\section{LA MUESTRA DE DATOS}

Con respecto a las fuentes de información con posibilidad de ser estudiadas, se ha realizado una observación de todos los materiales susceptibles de analizarse y se detecta gran cantidad de datos, se encontraron tesis o trabajos de grado, libros o textos editados y no editados, informes de investigación, comunicaciones y conferencias para congresos y publicaciones periódicas o seriadas en formato revista.

Se registraron los artículos publicados en revistas especializadas dejando de lado otras fuentes, esta decisión obedece a la posibilidad que las revistas ofrecen en cuanto que en sus contenidos se manifiestan los diversos tipos de producción posibles en Educación Física: artículos que sintetizan informes de investigación, textos tipo ensayo, reseñas, revisiones, traducciones, propuestas didácticas, entre otros; porque en las revistas se puede encontrar la potencial diversidad de las temáticas que componen la profesión, es decir que hay riqueza en la convergencia de variedad de posturas académicas y de diferentes tipos de formas de producir y presentar conocimientos.

La muestra se centra en las revistas porque en ellas se manifiestan muchas variables de la profesión en cuanto a sus temas de interés, sus procedimientos, sus resultados, sus reflexiones, sus opiniones y las ideas que circulan en el contexto donde se realiza el estudio. La muestra está compuesta por la totalidad de documentos de texto presentes en las publicasiones seriadas o periódicas especializadas en Educación física editadas entre el año 1990 y el 2006 en Colombia. Se aplico el instrumento a 1008 documentos (productos de conocimiento).

\begin{tabular}{|c|c|c|c|}
\hline \multicolumn{3}{|c|}{ Revista } & Documentos \\
\hline $\mathbf{A}$ & \multicolumn{2}{|c|}{ Revista Actividad Fisica y Desarrollo Humano } & 12 \\
\hline B & \multicolumn{2}{|l|}{ Revista Recrearte } & 10 \\
\hline $\mathbf{C}$ & \multicolumn{2}{|l|}{ Cuadernos de Ocio } & 24 \\
\hline D & \multicolumn{2}{|l|}{ Revista Con-Sentido } & 60 \\
\hline $\mathbf{E}$ & \multicolumn{2}{|c|}{$\begin{array}{l}\text { Revista Antioqueña de Medicina Deportiva y ciencias aplicadas al deporte y la } \\
\text { actividad fisica }\end{array}$} & 43 \\
\hline F & \multicolumn{2}{|c|}{ Revista Educación fisica y recreación } & 102 \\
\hline $\mathbf{G}$ & \multicolumn{2}{|c|}{ Revista Lúdica-Pedagógica } & 101 \\
\hline $\mathbf{H}$ & \multicolumn{2}{|c|}{ Revista Educación fisica y deporte } & 145 \\
\hline I & \multicolumn{2}{|l|}{ Revista digital Red-deporte } & 4 \\
\hline $\mathbf{J}$ & \multicolumn{2}{|l|}{ Revista Corpus 2000} & 5 \\
\hline \multirow[t]{2}{*}{$\mathbf{L}$} & \multicolumn{2}{|l|}{ Revista Kinesis } & 502 \\
\hline & \multicolumn{2}{|r|}{ Total } & 1008 \\
\hline \multicolumn{4}{|c|}{ Revistas descartadas } \\
\hline M & Cuademos de Ocio y Sociedad & \multirow{3}{*}{\multicolumn{2}{|c|}{$\begin{array}{l}\text { Ediciones fuera del límite temporal establecido para esta } \\
\text { investigación. }\end{array}$}} \\
\hline $\mathrm{N}$ & Revista Ímpetus & & \\
\hline 0 & Revista digital Corporeizando & & \\
\hline $\mathbf{P}$ & Revista Des-Encuentros & \multicolumn{2}{|c|}{$\begin{array}{l}\text { Excluida porque sus contenidos no son especializados en } \\
\text { Educación fisica. }\end{array}$} \\
\hline
\end{tabular}

Figura 2. La muestra de datos. 
En el caso de la muestra que corresponde a la modalidad de las Entrevistas en Profundidad, el universo de datos está constituido por los relatos orales producidos por 8 informantes que son actores protagónicos del campo profesional de la Educación física, se eligieron en función de las temáticas identificadas y que emergen del análisis documental.

\section{LOS HALLAZGOS}

Sólo presentan datos descriptivos y un análisis básico de la producción bibliográfica, los discursos orales serán presentados, analizados y detallados en otra publicación. Los rasgos académicos.

La idea de ciencia experimental ha ido instaurando unos rasgos para regular la credibilidad de los conocimientos y como consecuencia de esto las publicaciones progresivamente niegan difusión a ciertos documentos debido a que no cumplen con sus estándares, esta operación de discriminación configuran ámbitos que adquieren niveles, posiciones o dimensiones de reconocimiento y aceptación según sus destinatarios. El análisis permite diagnosticar el peso, el estado de prevalencias y de ausencia de unos rasgos frente a otros. Los aspectos que permitieron hacer una diferenciación son ( I ) textos que comunican Experiencias, acá fueron clasificados documentos que contienen la expresión escrita de vivencias; (2) Metodologías, bajo este rotulo se identifican los escritos que promueven herramientas, dispositivos, prácticas, procedimientos, instrucciones, modelos de gestión o protocolos de actuación; (3) Teóricos, este ítem señala los textos que ponen en circulación definiciones o conceptos; (4) Literarios, bajo este título se han clasificado documentos tipo poético, documentos con rasgos artísticos como poesías.

\begin{tabular}{|c|c|c|c|c|c|c|c|c|c|c|c|c|}
\hline \multirow{2}{*}{ Rasgos académicos } & \multicolumn{11}{|c|}{ Revistas } & \multirow{2}{*}{ Total } \\
\hline & $\mathbf{A}$ & B & C & D & $\mathbf{E}$ & $\mathbf{F}$ & G & $\mathbf{H}$ & I & J & $\mathbf{L}$ & \\
\hline 1 Experiencias,vivencias & 0 & 0 & 1 & 22 & 3 & 3 & 3 & 6 & 2 & 0 & 37 & 77 \\
\hline 2 Metodologfas-Modelos-desactuación & 8 & 1 & 0 & 6 & 3 & 27 & 15 & 44 & 1 & 0 & 94 & 199 \\
\hline s Conceptos, teorías, nociones & 4 & 8 & 20 & 46 & 36 & 73 & 81 & 73 & 3 & 4 & 370 & 718 \\
\hline 4 Literatura & 0 & 0 & 5 & 1 & 0 & 0 & 4 & 6 & 0 & 1 & 5 & 27 \\
\hline
\end{tabular}

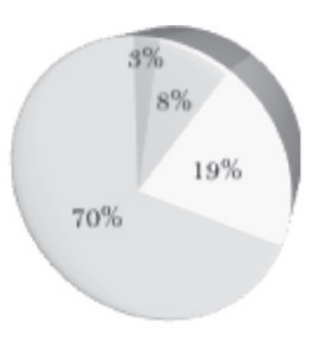

Figura 3. Datos numéricos que ilustran la identidad y los rasgos académicos y/o intelectuales de los documentos ${ }^{4}$.

Se observa que la mayoría de textos clasificados se ocupan de desarrollar, presentar o problematizar conceptos, ideas, nociones o teorías. Seguidamente con menor proporción los textos exponen, explican, reseñan o resaltan metodologías

4. Para ver los nombres de las revistas rotuladas de $A$ a $L$ ver cuadro en el apartado "La muestra de datos." 
de trabajo, de estudio o de investigación; a continuación le siguen los textos que hacen referencia a experiencias o vivencias y por último se encuentran en cantidad reducida los documentos de tipo literario.

\section{LAS FORMAS COMUNICATIVAS}

Otros factores que se logran identificar en la documentación recolectada, son los que se refieren a los patrones de presentación de los conocimientos. Al igual que en el anterior grupo de rasgos también las formas de comunicar conocimientos se acoplan a los requerimientos impuestos por las tendencias que gozan de mayor estatus o reconocimiento cultural. De este modo los problemas y temáticas profesionales se revisten de pautas de presentación, adoptan cierto tipo de guión estructural que los hacen más o menos adecuados para su lectura en comunidades específicas (administradores, políticos, profesores, investigadores, alumnado o público en general).

Según la destinación que se pretende los textos adquieren una forma, es decir, quienes los producen los dotan de una retórica que va dirigida a causar efectos en determinadas comunidades. Las revistas se valen, en muchos casos, de un tipo de caracterización de los documentos que permite su clasificación e incluso su valoración: reseñas (I) de textos y (2) de eventos, (3) avances, resultados o informes de investigación, (4) comunicados, manifiestos o declaraciones, (5) ensayos y (6) revisiones de tema o estados del arte.

\begin{tabular}{|c|c|c|c|c|c|c|c|c|c|c|c|c|c|}
\hline \multirow{2}{*}{\multicolumn{2}{|c|}{ Formas-comunicativas }} & \multicolumn{11}{|c|}{ Revistas } & \multirow{2}{*}{ Total } \\
\hline & & $\mathbf{A}$ & B & C & D & $\mathbf{E}$ & F & G & $\mathbf{H}$ & I & $\mathbf{J}$ & $\mathbf{L}$ & \\
\hline & Reseñas.de-textos & 0 & 0 & 0 & 4 & 0 & 1 & 0 & 0 & 0 & 0 & 15 & 20 \\
\hline 2 & Resenas-de-eventos & 0 & 0 & 0 & $s$ & 1 & 0 & 0 & o & 0 & 0 & 5 & 9 \\
\hline s & $\begin{array}{l}\text { Informes } \cdot \text { de } \cdot \text { investigación, } \cdot \text { resultados } \cdot \text { totales } \cdot 0 \\
\text { parciales }\end{array}$ & 5 & 4 & 0 & 11 & 15 & 14 & 29 & 61 & 1 & 2 & 39 & 181 \\
\hline 4 & Comunicados, manifiestos, declaraciones & 0 & 0 & 1 & 0 & 1 & 4 & 1 & 0 & 0 & 0 & 7 & 14 \\
\hline 5 & Ensayos & 6 & 4 & 24 & 46 & 14 & 71 & 54 & 75 & $\mathbf{s}$ & $s$ & 989 & 689 \\
\hline 6 & Revisión-de-temas,-estados-del-arte & 1 & 0 & 0 & 0 & 12 & 0 & 0 & 0 & 0 & 0 & 3 & 16 \\
\hline
\end{tabular}

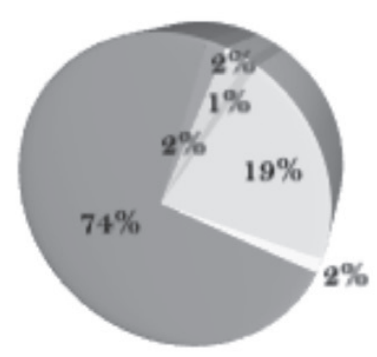

Figura 4. Resultados de la clasificación de los documentos a partir de su forma comunicativa. 
Los registros indican que la producción en su gran mayoría se presenta en el formato del ensayo, los documentos que poseen la tipología de redacción propia de la investigación están en segunda posición, las reseñas de texto y de eventos tienen escasa presencia en la muestra analizada, igualmente los comunicados, las revisiones de temas o estados del arte y los manifiestos se presentan como formas de comunicación poco usadas.

Cabe anotar que con respecto al patrón más empleado para la presentación de conocimientos, se ha considerado el ensayo como el tipo de escrito académico que permite la exposición ágil de investigaciones, opiniones, ideas; algunos autores consideran que por su versatilidad gozan de facilidad para publicación, obtiene un mayor número de lectores y produce un efecto más directo (VARGAS ACUÑA, 1996). Estas podrían ser algunas de las razones por las cuales los profesionales lo empleen como forma comunicativa. Por lo que se refiere al código que presenta, en segundo lugar, mayor difusión en la muestra codificada y que lleva por título "Informes de investigación, resultados totales o parciales", se observa que hay incrementos importantes al final del período analizado lo que puede corresponder con varios eventos que en la década de los 90's empiezan a influir en la profesión en Colombia, uno es la influencia de la implementación del sistema nacional de ciencia y tecnología -Colciencias- y la incorporación de requerimientos (como el formato de artículo científico, este tipo de exigencias privilegia y favorece claramente los contenidos provenientes de investigaciones) para publicar en las revistas.

\section{LOS ESCENARIOS ALUDIDOS}

La fuente documental es portadora de información que posibilita la asociación con un escenario o espacio de interés profesional, es posible buscar los signos y las señales explícitas que el documento contiene acerca de los lugares que están circulando, que son objeto de problematización, de estudio y tratamiento en los productos académicos de la Educación física. Este foco de observación puede tener utilidad para comprender poderes e intereses que se juegan en el campo profesional, pues las intenciones expresadas en los documentos analizados hacen posible su comprensión y aproximación a determinados centros de desempeño profesional.

Hay una amalgama de saberes, ciencias y profesiones que interactúan, condicionan y encauzan los recursos de las personas que se ocupan de la Educación física y todo ello influye para que la producción de prácticas y discursos de conocimiento se dirija a determinados escenarios. ¿Cuáles son los escenarios? I) Los educativos en general, 2) los de educación universitaria, 3) los de educación primaria y preescolar, 4) los de educación secundaria y media técnica, 5) los recreativos, 6) los deportivos, 
7) los clínico-médicos o terapéuticos, 8) los preventivos, de salud ocupacional o salud pública, 9) los de regulación y formación de profesionales y, 10) los de interés general o de la vida cotidiana.

\begin{tabular}{|c|c|c|c|c|c|c|c|c|c|c|c|c|c|}
\hline \multirow{2}{*}{\multicolumn{2}{|c|}{ Escenarios-aludidos }} & \multicolumn{11}{|c|}{ Revistas } & \multirow{2}{*}{ Total } \\
\hline & & A & B & $\mathrm{C}$ & $\mathrm{D}$ & $\mathrm{E}$ & $\mathrm{F}$ & G & $\mathrm{H}$ & I & $\mathrm{J}$ & $\mathrm{L}$ & \\
\hline 1 & Educativo-general & 1 & 1 & 11 & 40 & 1 & 32 & 65 & 99 & 0 & 0 & 101 & 351 \\
\hline $\boldsymbol{q}$ & Académicos-Educación-Universitaria & 2 & 7 & 7 & 41 & 0 & 17 & 28 & 50 & 1 & 0 & 110 & 263 \\
\hline $\mathbf{s}$ & Educación·Primaria·y•preescolar & 0 & 0 & 0 & 0 & 1 & 1 & 2 & 1 & 0 & 0 & 16 & 21 \\
\hline 4 & Educaciónsecundaria‘y•media-técnica & 0 & 0 & 0 & 1 & 1 & 0 & 0 & 0 & 2 & 0 & $s$ & 7 \\
\hline 5 & Recreativo & 0 & 0 & 1 & 0 & 0 & 6 & 5 & 14 & 0 & 0 & $2 s$ & 49 \\
\hline 6 & Deportivo & 11 & 2 & 0 & 1 & $\$ 6$ & 47 & 28 & 47 & 2 & 5 & 312 & 491 \\
\hline 7 & Médico,cclínico,-terapéutico & 2 & 0 & 0 & 1 & 19 & 7 & 2 & 10 & 1 & 0 & 35 & 77 \\
\hline 8 & Médico-preventivo-salud-ocupacional-o-publica & 1 & 3 & 5 & 5 & 7 & 11 & 1 & 12 & 1 & 0 & 74 & 120 \\
\hline 9 & Regulación•y•formación-de-profesionales & 0 & 1 & 5 & 5 & 1 & 2 & 6 & 8 & 0 & 0 & 7 & 35 \\
\hline 10 & Vida-Cotidiana & 0 & 0 & 12 & 7 & 0 & 4 & 4 & 8 & 0 & 1 & 31 & 67 \\
\hline
\end{tabular}

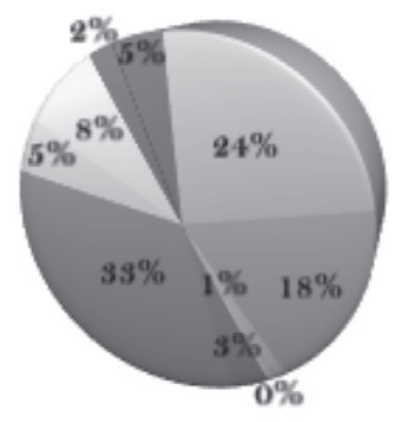

Figura 5. Escenarios implicados en la producción bibliográfica de la Educación Física.

La gran mayoría de documentos relaciona su discurso con los escenarios deportivos como protagonistas importantes de la producción. En orden decreciente se encuentran los espacios educativos, bajo este código se hallan textos en interacción con otros escenarios, por ejemplo, las relaciones entre salud-educación o formación, deporte-educación, hábitos-educación, etcétera; esta es una de las razones por las cuales en este código se concentran un gran número de escritos. Después se encuentran los temas de salud preventiva, luego de medicina clínica o terapéutica, seguidamente los que no definen claramente una institución sino que van a escenarios poco definidos (que acá se llaman de "interés general o para la vida cotidiana").

\section{LOS ACTORES SUGERIDOS}

Seguidamente la documentación se analizó buscando detectar o identificar los actores que potencialmente se benefician con los conocimientos que circulan 
en las revistas. Cabe recordar que una de las nociones de conocimiento que guía este análisis asume que el campo profesional es un espacio social que experimenta unas luchas y que el capital producido tiende a beneficiar espacios y actores según la visión que se imponga en la contienda; así que revisar cuales actores están implicados, resulta significativo e importante para comprender el campo profesional. Los textos codificados en el conjunto "actores" permiten identificar a los potenciales beneficiarios de los documentos. Estos son los principales: I) alumnado, 2) profesores, académicos o universitarios, 3) entrenadores y deportistas, 4) personal médico-sanitario, 5) colectivos y comunidades específicas, 6) investigadores, 7) administradores y gestores, y 8) comunidad en general.

\begin{tabular}{|c|c|c|c|c|c|c|c|c|c|c|c|c|c|}
\hline \multirow{2}{*}{\multicolumn{2}{|c|}{ Códigos }} & \multicolumn{11}{|c|}{ Revistas } & \multirow{2}{*}{ Total } \\
\hline & & A & B & $\mathrm{C}$ & $\mathrm{D}$ & $\mathrm{E}$ & $\mathrm{F}$ & G & $\mathrm{H}$ & I & $\mathrm{J}$ & $\mathrm{L}$ & \\
\hline 1 & Alumnos & 0 & 0 & 1 & 7 & 0 & $s$ & 6 & 1 & 0 & 0 & 4 & 22 \\
\hline$q$ & Profesores,académicos-o-universitarios & 1 & 5 & 8 & 47 & 2 & 51 & 74 & 109 & 1 & 0 & 178 & 476 \\
\hline $\mathbf{s}$ & Entrenadores-y.deportistas & 11 & 4 & 0 & 2 & \$4 & 49 & 31 & 54 & 4 & 5 & 934 & 528 \\
\hline 4 & Personal-médico-sanitario & 2 & 2 & 0 & $s$ & 25 & 17 & $s$ & 16 & 0 & 0 & 72 & 140 \\
\hline 5 & Colectivos,-comunidades & 0 & 0 & 2 & 1 & $s$ & 1 & 1 & 5 & 0 & 0 & 8 & 21 \\
\hline 6 & Investigadores & 0 & 0 & 0 & 40 & 0 & 19 & 14 & 18 & 0 & 0 & 10 & 96 \\
\hline 7 & Administradores, gestores & 1 & 1 & $s$ & 6 & 1 & 13 & 26 & 19 & 0 & 0 & 12 & 82 \\
\hline & Comunidaden.general & 0 & 0 & 19 & 14 & 1 & 18 & 6 & 16 & 0 & 1 & 38 & 113 \\
\hline
\end{tabular}

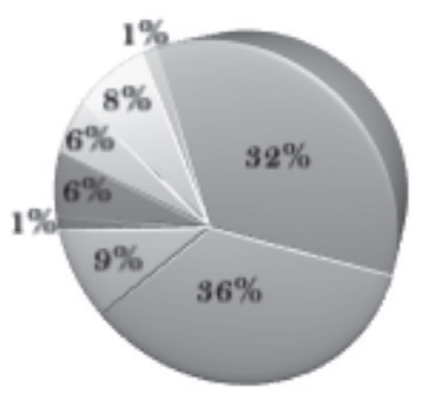

Figura 6. Resultado del registro de documentos en conjunto de códigos actores.

Los hallazgos indican que los textos agrupados bajo el código entrenadores y deportistas son los que predominan, seguidos por los documentos que tienen en consideración a los profesores universitarios, otro grupo importante de documentos estaría agrupado bajo el código investigadores, en seguida se encuentran los textos que involucran a la comunidad en general, a continuación están ubicados los textos que atienden a "los administradores", luego están en número menor el grupo que remite al personal médico-sanitario. Presentan menor cantidad de documentos los actores como alumnos, colectivos o comunidades específicas, esto refleja el estado de la comunicación a estos posibles beneficiarios de la Educación física. 


\section{LAS TEMÁTICAS QUE CONFIGURAN EL CAMPO PROFESIONAL DE LA EDUCACIÓN FÍSICA EN COLOMBIA}

Corresponde ahora hacer frente a la pregunta: ¿Cuáles son los problemas, asuntos y temas que configuran la producción de conocimiento en la Educación física en Colombia? Las relaciones, las presiones, las luchas, los roles asumidos por los actores, las interacciones de las instituciones implicadas se manifiestan en el campo en temáticas y problemas que van delimitando y configurando una geografía propia al campo profesional. Se identificaron en las publicaciones analizadas 39 sentidos que puede tener la Educación Física.

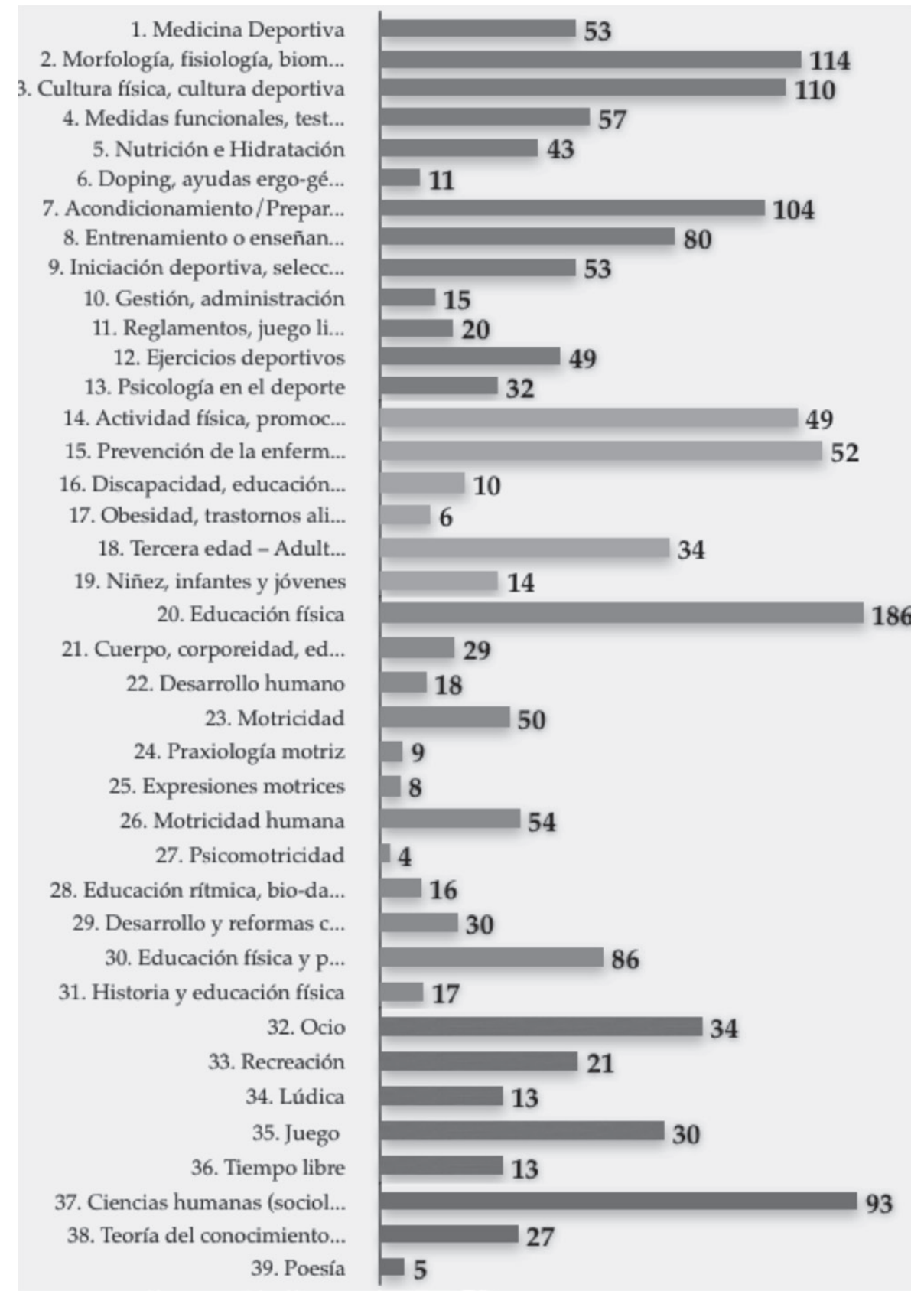

Figura 7. Presencia de temáticas en la muestra analizada. 
Los documentos analizados presentan en algunos casos discursos tendientes a la contraposición, esta situación sumada a la gran diversidad permite interpretar interacciones complejas de saberes, ciencias y disciplinas, relaciones que en se convierten en algunos casos de complementariedad y en otros de oposición. Las primeras se pueden interpretar por ejemplo en los productos que retoman principios teóricos o métodos médicos para contextualizarlos en un escenario y con actores de la Educación física. Las últimas son comprensibles en algunos documentos donde se proponen alternativas teóricas o metodológicas a las heredadas de otras campos, ciencias o saberes; es decir, se resaltan las bondades de una nueva propuesta y se hace énfasis en las carencias de sus opositoras. Este fenómeno de interacción tiene implícita la disputa por los posicionamientos en el campo profesional.

Los autores de los artículos estudiados ponen en juego un capital académico y de conocimiento. Este acto garantiza la reproducción, la evolución y la distinción en el sistema de relaciones sociales y de poder que son constitutivas del campo profesional. Y tiene como objetivo asegurar, promover e imponer una parcela en el espacio del campo.

Los campos son el lugar de relaciones de fuerzas, que implican tendencias inmanentes, probabilidades objetivas. Un campo no se orienta en absoluto por el azar. En él, no todo es igualmente posible o imposible en cada momento (BOURDIEU, 2000, p.80).

Estamos ante un fenómeno expansivo que es cada vez más contrario al ideal de una Educación física universal, con principios uniformes, con una única definición o con unos solos procedimientos de actuación. La diversidad y la interacción entre las temáticas manifestadas en el campo profesional conforman un estado de tensiones y de permanente complejidad del campo. Ahora, bien: icómo leer, interpretar y comprender la variedad de temáticas y problemas evidenciados? El procedimiento metodológico que posibilita avanzar en este sentido produce lo que acá se ha llamado Unidad Significativa ${ }^{5}$, conviene recordar que estos dispositivos de análisis emergen en parte del estudio de la fuente bibliográfica y en otra parte de las entrevistas en profundidad.

\section{UNIDADES SIGNIFICATIVAS QUE CONFORMAN EL CAMPO PROFESIONAL DE LA EDUCACIÓN FÍSICA EN COLOMBIA.}

El análisis de la producción de conocimiento permitió conocer los rasgos académicos, evidenciar las formas comunicativas empleadas, identificar los escenarios

5. Ver nota número 4 
profesionales implicados, diferenciar los actores y rastrear la diversidad de asuntos, temáticas, y problemáticas que ocupan la atención de los autores que participan del campo profesional. A continuación se presentan las Unidades Significativas que reflejan conjuntos de problemas, de propósitos, de objetos incluso; cada una ostenta identidad, independencia y por lo general manifiestan exclusión y diferenciación.

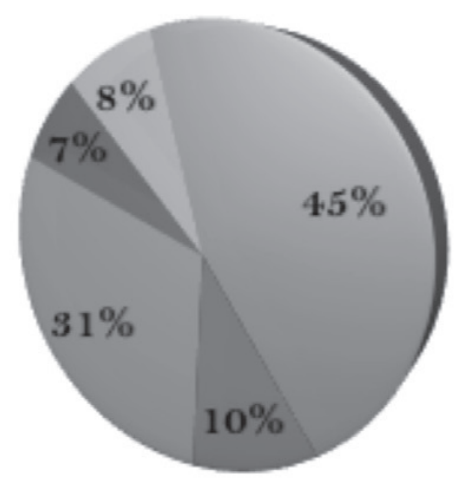

US1 Deporte, rendimiento y competición $=741$

US2 Act. física, promoción y prevención en salud $=165$

USs Escuela, educación y pedagogía $=507$

US4 Ocio, recreación, lúdica, juego $=111$

Otros $=125$

Figura 8. El campo profesional de la Educación física descrito en cifras.

\section{USI: EL DEPORTE, EL RENDIMIENTO Y LA COMPETICIÓN}

El $45 \%$ de los documentos analizados corresponde a discursos y prácticas que toman en consideración conocimientos acerca del desarrollo físico o del movimiento en función del rendimiento deportivo, la competición y los altos logros. Se refleja una tendencia, se presentan procedimientos y metodologías de trabajo diferenciables con claridad de otras unidades. Frente a los propósitos que persigue este grupo considero relevante la narración del informante quien en la entrevista en profundidad destaca como una de sus proyecciones y perspectivas de búsqueda profesional la siguiente:

Pedro $^{6}$ : Los problemas que intento abordar en mis investigaciones tienen que ver sobre todo con el alto rendimiento, desde que inicie mi carrera he estado interesado en el tema. Tengo I 6 años trabajando en esto de la preparación atlética y percibo que la Educación física ha avanzado mucho últimamente porque nos llaman de ligas deportivas a dar conferencias a los entrenadores que están iniciando temporada con deportista que compiten en todos los ámbitos. (...) normalmente participo en investigaciones que tratan de averiguar cómo mejorar el rendimiento en los deportistas élite del patinaje. (...) veo que lo que hago tiene mucha utilidad para el país, porque en cada triunfo que obtienen nuestros deportistas, siento que hay aportes, veo que en algo he ayudado para que se concreten los triunfos.

6. Los nombres de los informantes han sido modificados. 


\section{US2: LA ACTIVIDAD FÍSICA PARA LA PROMOCIÓN Y LA PREVENCIÓN EN LA SALUD}

Un conjunto de escritos destaca porque sus contenidos atienden la búsqueda, el mantenimiento, la promoción o la recuperación de la salud. Estos textos constituyen el $10 \%$ de la totalidad de la muestra analizada. Las temáticas que acá han sido agrupadas suelen encontrarse asociadas de algún modo con el deporte, esto tiene que ver con sus fuentes teóricas y conceptuales que proceden de las ciencias o saberes biomédicos; no obstante he optado por reunirlas en un conjunto diferente buscando conjugar los discursos y las prácticas de conocimientos relacionados con las actividades físicas que están en función del mantenimiento, la promoción o la recuperación de la salud.

Un actor que profesa esta perspectiva en su relato explica el propósito de su quehacer, retomo un fragmento de conversación que deja ver la idea general que permite agrupar trabajos en este conjunto:

Teresa: tengo una maestría en salud colectiva, en mi trabajo de grado estudie los factores de vulnerabilidad que pueden afectar una comunidad de tercera edad, y busco contrarrestarlos por medio de un plan experimental de actividad física.

\section{US3: LA PEDAGOGÍA, LA EDUCACIÓN Y LA ESCUELA.}

En la presente unidad significativa he reunido las temáticas asociadas con los escenarios escolares que tienen por objetivo la formación corporal de las personas. Este conjunto se caracteriza porque los elementos que lo componen exponen el interés por atender los problemas educativos o pedagógicos, las temáticas incluidas plantean como escenarios de actuación profesional los espacios que tienen lugar en el sistema educativo. Se han agrupado las temáticas que están en función de educar el cuerpo, de desarrollar didácticas de la motricidad o el movimiento, de problematizar y controvertir las múltiples teorías acerca de la Educación física.

Una informante protagonista de esta porción de productos me ha dado pistas con su relato para la clasificación:

Alba: Una cosa muy importante, que en mi caso he venido promoviendo, es lo que te decía que quería exponer en la Educación física, era cómo desde las Expresiones Motrices podíamos hacer una propuesta para la Escuela, no para la Educación física sino para la escuela y cómo desde ahí se han generado lo que yo he llamado, mal llamado tal vez por parte de expertos, esto: Principios didácticos entorno a una pedagogía de la motricidad o del cuerpo. 
El 7\% de los documentos desarrolla temáticas que tiene como propósito el estudio, el tratamiento y el desarrollo de discursos que atienden a la diversión y el esparcimiento. Son textos que contienen discursos y conocimientos acerca del ocio, el tiempo libre, la recreación, la lúdica, el juego. Acá se han agrupado trabajos que guardan una interdependencia en función de temáticas que se presentan al lector como diferentes del deporte, la salud, la educación y, en cambio, se despliegan en los marcos de una ideología y de unas operaciones que buscan clara diferenciación de las otras unidades significativas que se interpretan en el campo.

Pepe: es posible tomar "el ocio" como un objeto de estudio independiente de la Educación física, el deporte o la recreación porque acá estamos hablando de asuntos que tienen que ver con el uso que hace el ser humano de su tiempo libre. Acá se puede estudiar por ejemplo las connotaciones que tiene el tiempo libre para un obrero, para un niño, para una ama de casa. Y luego hacer propuestas para cada connotación y significación.

\section{DISCUSIÓN Y CONCLUSIONES}

Entre las principales reflexiones que ha generado el proceso de problematización, registro y análisis acerca de las cuestiones propuestas al inicio de esta investigación, una fundamental es que este trabajo constituye un intento por abordar el cuestionamiento acerca de la identidad del campo profesional de la Educación física, tomando como referente el estudio histórico-social de los productos generados por los actores que acá interactúan.

Los propósitos formulados para identificar las temáticas que conforman el campo profesional en cuestión quedan cubiertos al hallar y clasificar 39 sentidos (temáticas) que los autores ponen en circulación en la Educación Física; debe añadirse un nivel más profundo de análisis a estos sentidos si se quiere alcanzar un grado mayor de comprensión de la Educación física, pero dado que el propósito acá esbozado era su identificación se considera que se supera esta expectativa e incluso se avanza hasta la interpretación de lo que operativamente se ha llamado Unidades Significativas, fundamentadas tanto en los datos bibliográficos como en las propias voces de los actores protagonistas

Emergen cuatro elementos o rasgos diferenciadores de la producción que presentan en primer lugar y mayoritariamente artículos con trazos teóricos o conceptuales debido a que la gran mayoría de textos expone fundamentaciones, fuentes teóricas, líneas académicas, tendencias de conocimiento. Otros rasgos presentes corresponden a la exposición de metodologías, herramientas, dispositivos, procedimientos, 
modelos de gestión o protocolos de actuación. También se encuentran presentes en menor medida trazos de tipo experiencial y artísticos (literatura con matices poéticos). El predominio de un rasgo nos da pistas acerca de las preocupaciones de los productores de conocimiento en la Educación Física, los dos señales con mayor presencia (teorías y metodologías) dan cuenta de una dinámica de campo que busca gestar una madurez de capital académico, sin embargo la presencia de otros tipos de documentos (literarios-artísticos y experienciales) manifiesta una estructura de campo que conforma un sistema de interacciones entre una pluralidad de instancias, de estilos, de tendencias (BOURDIEU, 2002b)

De otro lado, encontramos las formas comunicativas que están expuestas en la producción y que guardando coherencia con los hallazgos acerca de los rasgos, dejan ver que el formato que predomina es el de los ensayos, seguido por los informes de investigación que se manifiestan en una importante cantidad y sin embargo, alejados de los primeros; y en pocas cantidades aparecen las reseñas, los comunicados y los estados de arte. La segunda forma comunicativa presente se refiere a los informes de investigación que especialmente presentan un incremento progresivo en los años finales del periodo analizado; esto podría obedecer a que apara el inicio del siglo XXl ya existe en Colombia un importante tejido de grupos e individuos dedicados a la investigación formal y en consecuencia revisten sus productos de los formatos de mayor circulación.

Con respecto a los escenarios y actores aludidos e implicados en la producción, se expone y manifiesta de forma más directa la disputa por participar del campo profesional, esto se hace evidente en la diversidad y aparente contradicción de actores y escenarios que permite interpretar un fenómeno político de posicionamiento en la Educación física, bien podría ser caracterizado por la afirmación de Serres acerca de su visión del conocimiento "fenómenos inestables, fluidos, volátiles, sutiles y difíciles, sin abstracción, obligando a recoger infinitos datos, evidentemente privados de regularidades sencillas, repletos de incertidumbres[...]" (SERRES, 1994, p. 92).

A los cinco elementos con los que acá se han identificado la producción de la Educación física (rasgos académicos, forma comunicativa, actores, escenarios, temáticas) ahora hay que adicionar la interpretación que la conjugación de los anteriores produce: las Unidades Significativas que muestran como la producción de conocimientos en el campo profesional se enfoca en un 45\% al deporte, el rendimiento y la competición, en un $10 \%$ a la actividad física, la promoción y la prevención en salud, el $31 \%$ de los datos se enfoca a la Escuela, la educación y la pedagogía, el 7\% de la información alude al ocio, la recreación, la lúdica y el juego y, finalmente, el $8 \%$ de documentos esta en función de otras temáticas difícilmente asociables al campo pero hacen presencia en este.

Además de estos resultados la investigación se perfila hacía la profundización del análisis en las Unidades Significativas, este dispositivo de indagación posibilita conectar todos los aspectos hasta acá tratados y cruzarlos, arrojando argumentos 
acerca de los criterios que permiten diferenciar y delimitar cada una de las Unidades que cruzadas con la noción Bourdesiana, han permitido trazar un dibujo interpretativo del campo profesional de la Educación Física, y que ganado complejidad en la medida que se interrelacionan los datos de escenarios y destinatarios con los propósitos y se comprenden los dominios que configuran las posiciones y la estructura del campo en cuestión; este último análisis referido esta aún en marcha y pendiente de publicarse.

\section{Análise parcial da produção acadêmica na área profissional de Educação} Física na Colômbia

RESUMO: O artigo apresenta a evolução das pesquisas sobre a produção dos acadêmicos de Educação Física na Colômbia, entre 1990 e 2006, onde são identificadas as características acadêmicas, os meios comunicação, os atores e as etapas envolvidas neste campo profissional. Foi implementado como métodos de análise do discurso documental e entrevistas em profundidade, abordando a desafio para a identidade profissional e a interação de conhecimentos, teorias e métodos que modelam e constituem esta parcela de acadêmicos.

PALAVRAS-CHAVE: Educação Física; treinamento de campo; a identidade profissional; produção acadêmica.

\section{A partial analysis of academic output in the professional field Physical Education in Colombia.}

ABSTRACT: The article presents developments in research on scholarly productivity in physical education in Colombia between 1990 and 2006, in that the academic characteristics, the means of communication, the actors and scenes implied in this professional field are identified. Was implemented as methods of discourse analysis documentary and in-depth interviews by approaching the question of professional identity and interaction of knowledge, theories, methods and knowledge that form and constitute this academic parcel.

KEYWORDS: Physical education; professional field; professional identity; academic output.

\section{REFERENCIAS}

BARBERO GONZÁLEZ, J. I. Cultura corporal: itenemos algo que decir desde la educación física? ágora para la educación física, Valladolid, n. I, p. I 8-36, sept. 2001.

BOURDIEU, P. Los usos sociales de la ciencia. Buenos aires: Nueva Visión, 2000. La lección sobre la lección. Barcelona: Anagrama, 2002a.

BOURDIEU, P. Campo de poder, campo intelectual. Tucumán: Montressor, 2002b.

BOURDIEU, P. El oficio de científico. Barcelona: Anagrama, 2003.

BRACHT, V. Educación física y aprendizaje social: educación física/ciencia del deporte: iqué ciencia es esa?. Córdoba: Vélez Sársfield, 1996. 
CARRASCO, J.; CALDERERO HERNÁNDEZ, J. Aprendo a investigar en educación. Madrid: Rialp, 2007.

CRISORIO, R. Educación física e identidad: conocimiento, saber y verdad. In: BRACHTV. y . La educación física en Argentina y Brasil: identidad, desafíos y perspectivas.

La Plata: Al Margen, 2003. p. 21 -38.

DEVÍS, J. D. et al. Las revistas científico-técnicas españolas de las ciencias de la actividad física y el deporte: inventario y análisis de la calidad de contenido y difusión. Revista Española de Documentación Científica, Madrid, v. 26, p. 177-190, marzo 2003.

FLECK, L. La génesis y el desarrollo de un hecho científico: introducción a la teoría del estilo de pensamiento y del colectivo de pensamiento. Madrid: Alianza, 1986.

FERNÁNDEZ-BALBOA, J.-M.; MUROS, B. The hegemonic triumvirate-idologies, discurses, and habitus in sport and physical education: implications and suggestions. Quest, n. 58, 2006, p. 197-221.

FERREIRA, A. L. et al. Revista de educação física: ciclo de vida, seção unidade de doutrina e lição de educação física ( | 932-2002). Movimento, Porto Alegre, n. 9, p. 9| - I | 8, jan./abr., 2003.

GALLO CADAVID, L. E. Cuatro hermenéuticas que han marcado la Educación Física en Colombia. In: CHAVERRA FERNÁNDEZ, B. E.; URIBE PAREJA, I. D. (Eds.), Aproximaciones epistemológicas y pedagógicas a la Educación Física: un campo en construcción. Medellín: Funámbulos, 2007. p. 45-69

KUHN, T. S. La estructura de las revoluciones científicas. México: Fondo de Cultura Económica, 2004.

MANNHEIM, K. Ideología y utopía. Madrid: Fondo de Cultura Económica, 1987.

MARTÍN-BARBERO, J. La crisis de las profesiones en la sociedad del conocimiento. Nómadas, Bogotá, v. |6, p. |77-|8|, abr. 2002.

MARTÍNEZ, N. La producción intelectual del licenciado en educación física entre el paredón y la palestra. Lúdica Pedagógica, Bogotá, n. 2, p. 37-40, enero 1996.

4, p. 15-10, enero, 2000

El profesional de educación física iquién es? Lúdica Pedagógica, Bogotá, n.

.; OLIVEROS, D. La investigación de los profesores en la facultad de educación física de la UPN: una aproximación conceptual sobre los resultados. Lúdica Pedagógica, Bogotá, n. 7, p. 19-27, enero, 2002.

MARTÍNEZ, E.; VARGAS, M. La investigación sobre la educación superior en colombia: un estado del arte. Bogotá: ICFES, 2002.

OLIVE, L. Los desafíos de la sociedad del conocimiento: cultura científico-tecnológica, diversidad cultural y exclusión. Revista Científica de Información y Comunicación, Sevilla, n. 3, p. 29-5I, 2006. 
REVERTER, J.; IZQUIERDO M. Estudio comparativo de tres revistas españolas de educación física y deportes. Apunts: Educación Física y Deportes, Barcelona, n. 89, p. I02-109, agosto 2007. Disponível em: <http://articulos-apunts.edittec.com/89/es/089_ I02-109ES.pdf>. Acesso em: 15 feb. 2010.

ROSSI, P. Las arañas y las hormigas: una apología a la historia de las ciencias. Barcelona: Editorial Critica, 1990.

RUIZ OLABUÉNAGA, J. I. Metodología de la investigación cualitativa. 4. ed. Bilbao: Universidad de Deusto, 2003.

SERRES, M. Atlas. Madrid: Cátedra, 1994.

VALENCIANO VALCÁRCEL, J. Calidad y producción científica de las revistas españolas de ciencias de la actividad física y el deporte. Valencia: Universitat de València, 2007.

VAREA, V. Educación Física y la producción de conocimiento: el caso de EFDeportes.com. Lecturas: Educación Física y Deportes, Buenos Aires n. 15, p. 01 -0 I, oct. 2010.

VARGAS ACUÑA, G. Un concepto de ensayo. Concurso de ensayo. 1996. Disponível em: http://www.cientec.or.cr/concurso2/concepto.html. Acesso em: 05 mayo 2009.

VICENTE PEDRAZ, M. Creencias pedagógicas, desubjetivación del cuerpo y dominación cultural en la Educación Física Escolar. Revista Brasileira de Ciências do Esporte, Florianópolis, v. 3 I, n. I, p. 109-129, set. 2009.

VILLAMÓN HERRERA, M. et al. Análisis de la visibilidad de las revistas científico-técnicas españolas de ciencias de la actividad física y el deporte. Revista de Psicología del Deporte, Palma, v. |4, p.253-267, oct. 2005.

VILLAMÓN HERRERA, M. et al. Análisis de las 'instrucciones para autores' de las revistas españolas de ciencias de la actividad física y el deporte. Motricidad: revista de ciencias de la actividad física y del deporte, Cáceres, n. 16, p. I33-150, jun. 2006.

WIEDER, R. E. (Coord.). Corporeidad, movimiento y educación física, 1992-2004. México: Consejo Mexicano de Investigación Educativa, 2007a. (Estudios conceptuales, n. I2, t. I) Corporeidad, movimiento y educación física, 1992-2004. México: Consejo Mexicano de Investigación Educativa, 2007b. (Estudios cuali-cuantitativos, n. 12, t. 2).

Recebido: 13 out 2010 Aprovado: 12 jun 2011

Endereço para correspondência: León J. Urrego D. Kra 75 No. 65-87 Barrio San German Ciudadela de Robledo U. de A. Bloque 45- I0 I Medellín-Antioquia-Colombia CP 050034 